\title{
Guidance for programmatic management of latent tuberculosis infection in the European Union/European Economic Area
}

\author{
Senia Rosales-Klintz ${ }^{1}$, Judith Bruchfeld ${ }^{2,3}$, Walter Haas ${ }^{4}$, Einar Heldal ${ }^{5}$, \\ Rein M.G.J. Houben ${ }^{6,7}$, Femke van Kessel ${ }^{8}$, Mike Mandelbaum ${ }^{9}$, \\ Alberto Matteelli ${ }^{10}$, Giovanni Battista Migliori (11), Anouk Oordt-Speets ${ }^{8}$, \\ Ivan Solovic ${ }^{12}$, Martina Vašáková ${ }^{13}$, Suzanne Verver ${ }^{14}$, Sake J. de Vlas ${ }^{14}$, \\ Marije J.M. Vonk Noordegraaf-Schouten ${ }^{8}$, Gerard de Vries ${ }^{15,16}$, \\ Dominik Zenner ${ }^{17}$ and Marieke J. van der Werf (10)
}

\begin{abstract}
Affiliations: ${ }^{1}$ European Centre for Disease Prevention and Control, Stockholm, Sweden. ${ }^{2}$ Dept of Infectious Diseases, Karolinska Institute, Stockholm, Sweden. ${ }^{3}$ Division of Infectious Diseases, Karolinska University Hospital, Stockholm, Sweden. ${ }^{4}$ Dept of Infectious Disease Epidemiology, Robert Koch Institute, Berlin, Germany. ${ }^{5}$ Norwegian Institute of Public Health, Oslo, Norway. ${ }^{6}$ TB Modelling Group, TB Centre, London School of Hygiene and Tropical Medicine, London, UK. ${ }^{7}$ Dept of Infectious Disease Epidemiology, London School of Hygiene, London, UK. ${ }^{8}$ Pallas Health Research and Consultancy, Rotterdam, The Netherlands. ${ }^{9}$ TB Alert, Brighton, UK. ${ }^{10}$ University Dept of Infectious and Tropical Diseases, and WHO Collaborating Centre for TB/HIV Collaborative Activities and for the TB Elimination Strategy, University of Brescia, Brescia, Italy. ${ }^{11}$ Istituti Clinici Scientifici Maugeri IRCCS, Tradate, Italy. ${ }^{12}$ National Institute for TB, Lung Disease and Thoracic Surgery, Vysne Hagy, Catholic University Ruzomberok, Ruzomberok, Slovakia. ${ }^{13}$ Dept of Respiratory Medicine, 1st Faculty of Medicine, Charles University and Thomayer Hospital, Prague, Czech Republic. ${ }^{14} \mathrm{Dept}$ of Public Health, Erasmus MC, University Medical Center Rotterdam, Rotterdam, The Netherlands. ${ }^{15} \mathrm{KNCV}$ Tuberculosis Foundation, The Hague, The Netherlands. ${ }^{16}$ National Institute for Public Health and the Environment, Bilthoven, The Netherlands. ${ }^{17}$ Public Health England, London, UK.
\end{abstract}

Correspondence: Marieke J. van der Werf, European Centre for Disease Prevention and Control, Gustav III:S 40, 16973 Solna, Sweden. E-mail: Marieke.vanderWerfdecdc.europa.eu

@ERSpublications

Management of latent tuberculosis infection is crucial to end TB in low-incidence settings http://ow.ly/pEcD30mJ8zL

Cite this article as: Rosales-Klintz S, Bruchfeld J, Haas W, et al. Guidance for programmatic management of latent tuberculosis infection in the European Union/European Economic Area. Eur Respir J 2019; 53: 1802077 [https://doi.org/10.1183/13993003.02077-2018].

Worldwide efforts are being made to end tuberculosis (TB) by 2035 following the ambitions outlined in the World Health Organization (WHO) End TB strategy [1] and the United Nations Sustainable Development Goals [2]. Countries with a low incidence of TB, i.e. less than 10 incident cases per 100000 population per year, should strive for TB elimination [3]. To this end, timely detection and treatment of latent tuberculosis infection (LTBI) is an important intervention [3]. Currently, the existence and implementation of national strategies including public health interventions targeting LTBI is heterogeneous across the European Union/European Economic Area (EU/EEA) [4]. To support the EU/EEA countries with developing national policies, as well as the planning and implementation of programmatic management of LTBI into national strategies for TB control, the European Centre for Disease Prevention and Control (ECDC) conducted a comprehensive assessment of the available evidence and developed an evidence-based guidance [5]. The guidance, published in October 2018, elaborates on population-level 
measures for LTBI management tailored to the EU/EAA context and it is complementary to the WHO guidelines [6]. Here, we summarise the process that was followed to develop the guidance and we outline the key components proposed for programmatic management of LTBI, to inform European healthcare professionals.

The ECDC guidance includes four key areas: target risk groups for programmatic management of LTBI; diagnosis of LTBI; treatment of LTBI; and programmatic issues of LTBI management. The key areas and corresponding research questions were identified through consultation with experts [7]. Scientific evidence was collected through systematic literature reviews, with additional evidence derived from mathematical modelling and cost-effectiveness analyses [8-10], and the evidence was reviewed and appraised by an ad hoc scientific panel.

The systematic literature reviews collected available scientific evidence on target groups, diagnosis and treatment of LTBI, and programmatic issues, and were performed in collaboration with WHO [11, 12]. Additional steps for identification, collection and appraisal of relevant peer-reviewed and grey literature were conducted, as described in a detailed technical report [8]. The evidence showed an increased risk of becoming latently infected and/or progressing to active TB disease for people living with HIV, immunocompromised patients, close contacts of TB patients (risk of progression especially high in children), migrants, healthcare workers, prisoners and homeless people [11, 12]. Both the tuberculin skin test (TST) and the interferon- $\gamma$ release assays (IGRA) were regarded as suitable and cost-effective diagnostic tools $[8,11,12]$. Similarly, various treatment regimens showed good efficacy and cost-effectiveness. Short treatment regimens (i.e. less than 6-month treatment duration) had better adherence and completion rates $[8,13]$. Several interventions were shown to improve initiation, adherence and completion of LTBI treatment, including provision of monetary incentives to people who inject drugs; nurse-led community-based case management in homeless people, educational sessions with prison inmates and counsellor or peer-based social support $[8,14,15]$.

The deterministic mathematical model for TB transmission estimated the contribution of LTBI screening and treatment strategies on reducing TB transmission. Various at-risk populations were considered in the model, i.e. people who inject drugs, homeless people, prisoners and migrants from high TB incidence countries. The model was applied to data from four EU countries: the Netherlands, the Czech Republic, Portugal and Spain. Modelling results suggested that screening for and treatment of LTBI in prisoners or migrants from high-endemic countries at entry in the country, people who inject drugs and homeless people all result in a decrease in pulmonary TB incidence. The order of importance of each of these groups depends on the country. The mathematical model informed the assessment of cost-effectiveness of selected LTBI screening and treatment strategies. Across all at-risk populations considered, model results found that performing a TST and if positive an IGRA was the most cost-effective strategy for diagnosing LTBI. The cost-effectiveness analysis further suggested that LTBI screening for migrants at entry, LTBI screening for prisoners and LTBI screening for people who inject drugs/homeless people would all be cost-effective. The cost-effectiveness of screening and treatment of four other groups was also assessed using cohort model variants: travellers, healthcare workers, immunocompromised patients and TB contacts. LTBI screening and treatment of travellers and healthcare workers would only be cost-effective under unrealistically high levels of increased risk for transmission in these populations. For immunocompromised patients, LTBI screening and treatment would be cost-effective if they are part of a migrant population or native populations in European countries with a relatively high TB burden (i.e. more than 50 incident cases per 100000 population). For close contacts of active pulmonary TB patients, the modelling found LTBI screening to be cost-effective $[9,10]$, which is in line with existing field studies $[16,17]$.

Based on the assessment of the scientific evidence and the expert opinion of the ad hoc scientific panel, ECDC identified key components for implementation of programmatic management of LTBI (table 1). Target groups proposed to be prioritised for LTBI screening and treatment are: people living with HIV; immunocompromised persons (patients on anti-tumour necrosis factor- $\alpha$ treatment, patients preparing for transplantation, patients with end-stage renal diseases and/or preparing for dialysis); patients with silicosis; people with pulmonary fibrotic lesions; and contacts of infectious TB cases. Additional at-risk groups may be considered depending on the TB epidemiology. For diagnosing LTBI, both TST and IGRA or a combination can be used. Table 2 summarises practical considerations for the selection of testing methods, based on the expert opinion of the ad hoc scientific panel. For successful implementation, LTBI screening should be conceptualised as a comprehensive strategy that requires availability of and access to diagnostic tests, and the intention to provide LTBI treatment (if appropriate) and the implementation of interventions promoting the uptake and completion of LTBI screening procedures. For treatment of LTBI, the following regimens can be considered: isoniazid alone (for 6-9 months), rifampicin alone (for 3-4 months), isoniazid and rifapentine (once weekly for 12 weeks), and isoniazid and rifampicin (for 3-4 months) [13]. The selection of the most appropriate LTBI treatment regimen should be based on an 
TABLE 1 Summary of the European Centre for Disease Prevention and Control guidance on programmatic management of latent tuberculosis infection (LTBI) in the European Union and European Economic Area [5]

Key components

\section{Target groups}

Identification of groups at-risk of having LTBI and/or an increased risk of progressing to active TB

\section{Diagnosis of LTBI}

Definition of diagnostic approach for LTBI detection, including both the selection of diagnostic test(s) and the diagnostic algorithm most appropriate for each target group

\section{Treatment of LTBI}

Provision of LTBI treatment using treatment regimens that are effective and promote adherence and completion by different target groups

\section{Programmatic issues}

Implementation of patient-centred strategies for service delivery

Effective health education and communication with target groups and healthcare providers

Programme monitoring and evaluation
Public health measures

Prioritisation of target groups for LTBI screening:

- people living with HIV

- immunocompromised persons, (patients on anti-TNF- $\alpha$ treatment, patients preparing for transplantation, patients with end-stage renal diseases and/or preparing for dialysis)

- patients with silicosis

- people with pulmonary fibrotic lesions

- contacts of infectious TB cases

Implementation of comprehensive strategy including:

- use of tuberculin skin test and interferon- $\gamma$ release assays lalone or a combination) to diagnose LTBI

- availability of and accessibility to diagnostic tests

- intention to provide LTBI treatment (if appropriate)

- implementation of interventions promoting the uptake and completion of LTBI screening procedures

Selection of LTBI treatment regimen from the following treatment regimens based on an individual risk assessment:

- isoniazid alone (for 6-9 months)

- rifampicin alone (for 3-4 months)

- isoniazid and rifapentine (for 3 months)

- isoniazid and rifampicin (for 3-4 months)

Implementation of an integrated strategy including:

- material incentives and enablers

- counselling and education

- peer-based support

- culturally sensitive approaches

Implementation of a comprehensive educational programme aiming at increasing awareness of the importance of detecting and treating LTBI

Implementation of programme monitoring and evaluation including: - establishment of a case-based registry of TB contacts identified during routine contact investigations

- revision/development of data collection processes

- definition of performance indicators

- implementation of regular programme monitoring, aligned with global [1] and regional [18] monitoring and evaluation frameworks

TB: tuberculosis; TNF: tumour necrosis factor.

individual risk assessment [21, 22]. Patient-centred case management including provision of material incentives and enablers, counselling and education, peer-based support and culturally sensitive approaches can be considered as part of an integrated programmatic strategy for LTBI management.

Country-level implementation of the suggested public health measures will need to take into account the TB epidemiology in various risk groups, health system structure, resource allocation and political commitment. In-depth knowledge of the local epidemiological profile will facilitate the identification of at-risk groups to be prioritised for LTBI screening and treatment. In addition, provision of high-quality programmatic management of LTBI will benefit from a well-coordinated collaboration between different levels of the health system (i.e. local, regional and national) and linkages with other health programmes (e.g. HIV clinics). The healthcare work force will need to be made aware, with appropriate training as necessary, of new and updated national guidelines, procedures, and specific technical (i.e. administration and interpretation of diagnostic tests) and social (i.e. establishing rapport and providing psycho-social support) skills.

We acknowledge that physicians will be confronted with the challenge of assessing the patient-level risk and benefits while implementing these population-level public health activities [23, 24]. Educational 
TABLE 2 Considerations for selection of latent tuberculosis infection (LTBI) testing method

\begin{tabular}{|c|c|c|}
\hline Target group & Preferred test & Reason \\
\hline Children under 5 years of age & TST & $\begin{array}{l}\text { Children's immune system, difficulty of drawing blo } \\
\text { performance of IGRAs in young children }\end{array}$ \\
\hline $\begin{array}{l}\text { Vulnerable and hard-to-reach } \\
\text { populations }{ }^{\#}\end{array}$ & IGRA & No need for a second visit to read the test result \\
\hline $\begin{array}{l}\text { Immunocompromised patients } \\
\text { (including PLHIV) }\end{array}$ & $\begin{array}{l}\text { Combination of TST and } \\
\text { IGRA (parallel testing)" }\end{array}$ & $\begin{array}{l}\text { LTBI tests are less sensitive in immunocompromise } \\
\text { In order not to miss Mycobacterium tuberculosis-in } \\
\text { may face significant adverse health effects due to } \\
\text { approach is advisable }\end{array}$ \\
\hline Migrant populations & $\begin{array}{l}\text { IGRA or TST acceptable } \\
\text { (IGRA for large numbers) }\end{array}$ & No need for a second visit to read the IGRA result \\
\hline BCG-vaccinated people & IGRA & TST may be affected by prior vaccination with BCG \\
\hline
\end{tabular}

PLHIV: people living with HIV; BCG: bacille Calmette-Guérin; TST: tuberculin skin test; IGRA: interferon- $\gamma$ release assay; TB: tuberculosis.

\#: adults, young people and children whose social circumstances or lifestyle, or those or their parents or carers, make it difficult to recognise TB symptoms, access health services, self-administer treatment and attend regular healthcare appointments [19]; ๆ: after the initiation of antiretroviral treatment, repeated testing for LTBI may be considered for PLHIV previously known to have negative TST or IGRA results [20]. Reproduced from [5] with permission.

interventions and incentives for frontline healthcare workers may support them in making these assessments and help overcome provider-related barriers to access LTBI diagnosis and treatment [25]. We also acknowledge the necessity to understand health practitioners' perceptions of and attitudes towards LTBI management to tailor information and advice that aims to increase their adherence to national guidelines.

Similarly, patient-related barriers such as poor health literacy and barriers related to cultural background and/or language should be minimised $[26,27]$. Efforts can include implementation of patient-centred approaches that take into consideration the social context and provision of psychological, social and financial support to at-risk populations [19].

Monitoring and evaluation of the programmatic approach to LTBI management can pose a major challenge for a national TB control programme, but is important to tackle. We encourage EU/EEA member states to create or continue improving their LTBI surveillance systems, striving for data completeness and more accurate reporting of those eligible, tested and treated for LTBI. These efforts will contribute to quantify the country-specific cascade of care for LTBI, and help identify areas for adaptation and improvement of LTBI programmatic management [28].

Finally, implementation and scale-up of programmatic management of LTBI would benefit from the exchange of lessons learned and experiences gained. There are already some published examples from European settings that show the importance of documenting local or national experiences [29-31]. Operational research on the effectiveness and cost-effectiveness of implemented LTBI interventions could help us further our understanding of the actual impact of programmatic LTBI management.

Conflict of interest: S. Rosales-Klintz has nothing to disclose. J. Bruchfeld has nothing to disclose. W. Haas has nothing to disclose. E. Heldal has nothing to disclose. R.M.G.J. Houben has nothing to disclose. F. van Kessel reports receiving grants from the ECDC. M. Mandelbaum has nothing to disclose. A. Matteelli has nothing to disclose. G.B. Migliori has nothing to disclose. A. Oordt-Speets reports receiving grants from the ECDC. I. Solovic has nothing to disclose. M. Vašakova has nothing to disclose. S. Verver reports receiving grants from the ECDC. S.J. de Vlas reports receiving grants from the ECDC. M.J.M. Vonk Noordegraaf-Schouten reports receiving grants from the ECDC. G. de Vries has nothing to disclose. D. Zenner has nothing to disclose. M.J. van der Werf has nothing to disclose.

\section{References}

$1 \quad$ World Health Organization. Implementing the End TB strategy: the essentials. Geneva, WHO, 2015.

2 United Nations General Assembly. The Sustainable Development Goals, 2015. www.un.org/sustainabledevelopment/ sustainable-development-goals/ Date last accessed: April 7, 2017.

3 Lonnroth K, Migliori GB, Abubakar I, et al. Towards tuberculosis elimination: an action framework for low-incidence countries. Eur Respir J 2015; 45: 928-952.

4 Kunst H, Burman M, Arnesen TM, et al. Tuberculosis and latent tuberculous infection screening of migrants in Europe: comparative analysis of policies, surveillance systems and results. Int J Tuberc Lung Dis 2017; 21: 840-851. 
5 European Centre for Disease Prevention and Control. Programmatic management of latent tuberculosis infection in the European Union. Stockholm, ECDC, 2018.

6 World Health Organization. Latent tuberculosis infection: updated and consolidated guidelines for programmatic management. Geneva, WHO, 2018.

7 Sandgren A, Vonk Noordegraaf-Schouten JM, Oordt-Speets AM, et al. Identifying components for programmatic latent tuberculosis infection control in the European Union. Euro Surveill 2016; 21: 30325.

8 European Centre for Disease Prevention and Control. Review of reviews and guidelines on target groups, diagnosis, treatment and programmatic issues of latent tuberculosis control. Stockholm, ECDC, 2018.

9 European Centre for Disease Prevention and Control. Mathematical modelling of programmatic screening strategies for latent tuberculosis infection in countries with low tuberculosis incidence. Stockholm, ECDC, 2018. Available from: https://ecdc.europa.eu/sites/portal/files/documents/Technical-Report_LTBI_math_modelling.pdf

10 European Centre for Disease Prevention and Control. Cost-effectiveness analysis of programmatic screening for latent tuberculosis infection in the EU/EEA. Stockholm, ECDC, 2018. Available from: https://ecdc.europa.eu/sites/ portal/files/documents/LTBI\%20cost-effectiveness\%20report.pdf

11 World Health Organization. Evidence to decision framework. Appendix to the Guidelines on the management of latent tuberculosis infection. WHO/HTM/TB/2015.01. Geneva, World Health Organization, 2015.

12 Getahun H, Matteelli A, Abubakar I, et al. Management of latent Mycobacterium tuberculosis infection: WHO guidelines for low tuberculosis burden countries. Eur Respir J 2015; 46: 1563-1576.

13 Zenner D, Beer N, Harris RJ, et al. Treatment of latent tuberculosis infection: an updated network meta-analysis. Ann Intern Med 2017; 167: 248-255.

14 Sandgren A, Vonk Noordegraaf-Schouten M, van Kessel F, et al. Initiation and completion rates for latent tuberculosis infection treatment: a systematic review. BMC Infect Dis 2016; 16: 204.

15 Stuurman AL, Vonk Noordegraaf-Schouten M, van Kessel F, et al. Interventions for improving adherence to treatment for latent tuberculosis infection: a systematic review. BMC Infect Dis 2016; 16: 257.

16 Pareek M, Watson JP, Ormerod LP, et al. Screening of immigrants in the UK for imported latent tuberculosis: a multicentre cohort study and cost-effectiveness analysis. Lancet Infect Dis 2011; 11: 435-444.

17 Zenner D, Hafezi $\mathrm{H}$, Potter J, et al. Effectiveness and cost-effectiveness of screening migrants for active tuberculosis and latent tuberculous infection. Int J Tuberc Lung Dis 2017; 21: 965-976.

18 WHO Regional Office for Europe. Roadmap to implement the tuberculosis action plan for the WHO European Region 2016-2020. Copenhagen, World Health Organization, 2016.

19 European Centre for Disease Prevention and Control. Guidance on tuberculosis control in vulnerable and hard-to-reach populations. Stockholm, ECDC, 2016. Available from: https://ecdc.europa.eu/sites/portal/files/media/ en/publications/Publications/TB-guidance-interventions-vulnerable-groups.pdf

20 Centers for Disease Control and Prevention. Latent Tuberculosis Infection: A Guide for Primary Health Care Providers. Atlanta, CDC, 2013.

21 Sotgiu G, Matteelli A, Getahun $\mathrm{H}$, et al. Monitoring toxicity in individuals receiving treatment for latent tuberculosis infection: a systematic review versus expert opinion. Eur Respir J 2015; 45: 1170-1173.

22 Migliori GB, Sotgiu G, Rosales-Klintz S, et al. ERS/ECDC Statement: European Union standards for tuberculosis care, 2017 update. Eur Respir J 2018; 51: 1702678.

23 Dobler CC, Luu Q, Marks GB. What patient factors predict physicians' decision not to treat latent tuberculosis infection in tuberculosis contacts? PLOS ONE 2013; 8: e76552.

24 Evenblij K, Verbon A, van Leth F. Intention of physicians to implement guidelines for screening and treatment of latent tuberculosis infection in HIV-infected patients in The Netherlands: a mixed-method design. BMC Public Health 2016; 16: 915.

25 Atchison C, Zenner D, Barnett L, et al. Treating latent TB in primary care: a survey of enablers and barriers among UK General Practitioners. BMC Infect Dis 2015; 15: 331.

26 Dobler CC, Bosnic-Anticevich S, Armour CL. Physicians' perspectives on communication and decision making in clinical encounters for treatment of latent tuberculosis infection. ERJ Open Res 2018; 4.

27 Abarca Tomas B, Pell C, Bueno Cavanillas A, et al. Tuberculosis in migrant populations. A systematic review of the qualitative literature. PLoS ONE 2013; 8: e82440.

28 Alsdurf H, Hill PC, Matteelli A, et al. The cascade of care in diagnosis and treatment of latent tuberculosis infection: a systematic review and meta-analysis. Lancet Infect Dis 2016; 16: 1269-1278.

29 Loutet MG, Burman M, Jayasekera N, et al. National roll-out of latent tuberculosis testing and treatment for new migrants in England: a retrospective evaluation in a high-incidence area. Eur Respir J 2018; 51: 1701226.

30 De Vries G, van Hest R, Bakker M, et al. Policy and practice of programmatic management of latent tuberculosis infection in The Netherlands. J Clin Tuberc Other Mycobact Dis 2017; 7: 40-48.

31 Collin SM, de Vries G, Lonnroth K, et al. Tuberculosis in the European Union and European Economic Area: a survey of national tuberculosis programmes. Eur Respir J 2018; 52: 1801449. 